\title{
Pharmacogenetics to prevent maniac affective switching with treatment for bipolar disorder: CYP2D6
}

\author{
"...potential drug interactions need to be taken into account when \\ selective serotonin reuptake inhibitors or tricyclic antidepressants are \\ combined with other drugs metabolized by CYP2D6..."
}

First draft submitted: 16 June 2016; Accepted for publication: 16 June 2016; Published online: 29 July 2016

Keywords: adverse event $\bullet$ bipolar disorder $\bullet$ CYP2D6 $\bullet$ pharmacogenetics patients [4], however, the evidence regarding the likelihood that antidepressant treatment in individuals with BPD confers increased risk of TEAS has long been inconclusive. In previous clinical trials. the maniac switch episodes were similar between antidepressant and placebo groups [5]. Furthermore, there is no consensus whether antidepressants may be harmful or beneficial to BPD patients [6]. USA guidelines do not recommend antidepressant use in bipolar depression unless depression is severe, which contrasts European guidelines.

The propensity to mood switching in bipolar patients has been attributed to individual differences in the genetic make-up of patients. Differences in a patient's capacity to metabolize drugs could therefore contribute to some of the observed adverse events. We are therefore assessing the value of utilizing pharmacogenetic information to improve antidepressant drug therapy in BPD patients [7].

\section{Role of CYP2D6 in response to bipolar disorder treatment}

CYP2D6 is a major drug metabolizing enzyme and one of the most studied CYP enzymes. It is involved in the metabolism of approximately $25 \%$ of the most commonly used drugs [8]. Regarding drugs used for the treatment of bipolar disorder, CYP2D6 contributes to approximately half of all the drugs prescribed including antidepressants and antipsychotics. Also, there is evidence from animal models indicating that CYP2D6 is

\begin{abstract}
Almudena Sánchez-Martín
Department of Pharmacy, University Hospital of Salamanca, Salamanca, Spain and

IBSAL, Salamanca Institute for Biomedical Research, Salamanca, Spain

Santiago Sánchez-Iglesias IBSAL, Salamanca Institute for Biomedical Research, Salamanca, Spain and

Department of Psychiatry, University Hospital of Salamanca, Spain

Belén García-Berrocal IBSAL, Salamanca Institute for Biomedical Research, Salamanca, Spain and

Department of Clinical Biochemistry, University Hospital of Salamanca, Spain

\section{Carolina Lorenzo}

IBSAL, Salamanca Institute for

Biomedical Research, Salamanca, Spain and

Department of Psychiatry, University Hospital of Salamanca, Spain
\end{abstract}

\section{Andrea Gaedigk}

Division of Clinical Pharmacology, Toxicology \& Therapeutic Innovation, Children's Mercy Hospital, Kansas City, MO, USA

and

Department of Pediatrics, School of Medicine, University of Missouri-Kansas City, Kansas City, MO, USA

María Isidoro-García Author for correspondence: IBSAL, Salamanca Institute for Biomedical Research, Salamanca, Spain and

Department of Clinical Biochemistry, University Hospital of Salamanca, Spain and

Department of Medicine, University of Salamanca, Spain misidoro@usal.es

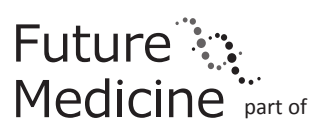
458 
involved in the biosynthesis of dopamine and 5-HT. Of note, CYP2D6 may be inhibited by a number of drugs that may further complicate complex medication schedules. For all these considerations and their involvement in clinical practice, it has been one of the most studied enzymes $[9,10]$.

\section{“...bipolar patients genotyped as CYP2D6 poor metabolizers suffered from maniac episodes after taking a drug that is metabolized by CYP2D6..."}

Over 100 allelic variants have been defined for CYP2D6 to date by the Human Cytochrome P450 Allele Nomenclature Committee [11]. Allele frequencies vary considerably among populations. Overviews of the CYP2D6 gene locus and phenotype prediction have been published by Gaedigk [12] and Hicks et al. [13] and genotype-based dosing guidelines for tricyclic antidepressants and SRRIs have been published by the Clinical Pharmacogenetics Implementation Consortium (CPIC) [13]. These guidelines also provide extensive information regarding allelic variation and frequency. Additional information regarding predicted phenotype (metabolizer status) has recently been published by Gaedigk et al. [14].

There are four defined phenotype categories: poor metabolizers (PMs), intermediate metabolizers (IMs), normal metabolizers (NMs) and ultra-rapid metabolizers (UMs). Based on allele frequencies, the PM phenotype is lowest in Asians and Oceania (0-2\%) followed by the Americas (0-8\%), Africans and AfricanAmericans (1-6\%) and Europeans (2-11\%) [15,16]. Ultrarapid metabolism on the other end of the extreme activity distribution also varies among world populations (0-22\%) [14].

Patients with bipolar disorders present with a maniac (or hypomaniac) episode or depressive episodes. Switching is a major risk factor during the treatment of bipolar depression. Recently, it has been reported that bipolar patients genotyped as CYP2DG PMs suffered from maniac episodes after taking a drug that is metabolized by CYP2D6 such as the selective serotonin reuptake inhibitors (SSRI) paroxetine or fluoxetine [7].

Moreover, it is also important to consider interactions with other drugs. In the case of patients with bipolar disorder, the majority is treated for other conditions as well and will likely take numerous drugs of which some may be CYP2D6 substrates or inhibitors and may lead to undesirable plasma levels in poor metabolizers [17]. Consequently, potential drug interactions need to be taken into account when SSRIs or tricyclic antidepressants are combined with other drugs metabolized by CYP2D6 [18]. Examples are paroxetine and fluoxetine, antidepressant inhibiting CYP2D6 with the potential to turn genotypic normal and intermediate metabolizers into PMs (a process also known as phenocopying) [19].

\section{Clinical application of CYP2D6 pharmacogenetic testing}

One of the main applications in clinical practice of pre-emptive CYP2D 6 genotyping is the identification of psychiatric patients with an increased risk of developing adverse effects. Patients with genotypes predicting poor or intermediate metabolizer status receiving CYP2D6-mediated drugs may develop an exaggerated response or toxicity. On the other end of the extreme, ultra-rapid metabolizers may not favorably respond, because they do not reach the target drug level.

Individualizing drug therapy (choice of drug and/or dosage) has the prospect of reaching target drug levels within a shorter period of time and avoiding potentially interfering drug combinations. Pharmacogenetic testing provides highly valuable information to guide initial treatment regimens as well as treatment adjustments of patients whose drugs 'did not work'. For BPD patients, genotype analysis could identify those patients with an increased risk of suffering a switching episode after initiating certain SSRI treatment. For patients with known genotype, the CPIC guideline recommends reducing the dose for SSRIs metabolized by CYP2D6 for PMs [13].In our experience alternative antidepressants whose metabolism does not depend on CYP2D6 such as escitalopram, sertraline or mirtazapine are alternative options. Of note, many SSRIs are metabolized by CYP2C19, another polymorphic enzyme. CYP2C19 genotype should therefore also be taken into consideration when choosing an appropriate alternative (see CPIC SSRI guideline).

Implementing pharmacogenetic testing into routine clinical practice may be challenging due to the cost of the test especially if not covered by insurance. However, the cost of pharmacogenetic testing may easily outweigh financial burden of repeat patient visits, adverse events, hospitalizations as well as patient safety, loss of quality of life of the patient and his/her family, loss of earnings, among others.

Other factors are without doubt contributing to drug response in BPD patients. However, the utilization of genotype-guided drug therapy, albeit not perfect, constitutes an important first step in the adoption of individualized drug therapy in this complex patient population.

\section{Conclusion \& future perspective}

Personalized medicine is opening important perspectives [20] and is increasingly embraced by clinicians. Information regarding its utility is urgently needed [21]. 
The determination of metabolizer status before treatment initiation would be most valuable to individualize therapy. Unfortunately, most of times genotyping is sought retrospectively 'a posteriori' to help explain adverse events or therapeutic failure. This approach, however, disadvantages patients with extreme phenotypes of receiving optimal care.

For these reasons, we consider that CYP2D6 genotyping could be very useful in the psychiatric setting especially in BPD treated with antidepressant. It provides important benefits in drug safety by identifying patients at risk to develop affective switching when

\section{References}

1 Salvadore G, Quiroz JA, Machado-Vieira R et al. The neurobiology of the switch process in bipolar disorder: a review. J. Clin. Psychiatry 71, 1488-1501 (2010).

2 Licht RW, Gijsman H, Nolen WA, Angst J. Are antidepressants safe in the treatment of bipolar depression? A critical evaluation of their potential risk to induce switch into mania or cycle acceleration. Acta Psychiatr. Scand. 118(5), 337-346 (2008).

3 Henry C, Sorbara F, Lacoste J, Gindre C, Leboyer M. Antidepressant-induced mania in bipolar patients: identification of risk factors. J. Clin. Psychiatry 62(4), 249-255 (2001).

4 Tamada RS, Lafer B. Manic episodes during antidepressant treatment in bipolar disorder. Rev. Bras. Psiquiatr. 25, 171-176 (2003).

5 Young AH, McElroy SL, Bauer M et al. A double-blind, placebo-controlled study of quetiapine and lithium monotherapy in adults in the acute phase of bipolar depression (EMBOLDEN I). J. Clin. Psychiatry 71, 150-162 (2010).

6 Vieta E, Locklear J, Gunther O et al. Treatment options for bipolar depression: a systematic review of randomized, controlled trials. J. Clin. Psychopharmacol. 30, 579-590 (2010).

7 Sánchez-Iglesias S, García-Solaesa V, García-Berrocal B et al. Role of pharmacogenetics in improving the safety of psychiatric care by predicting the potential risks of mania in CYP2D6 poor metabolizers diagnosed with bipolar disorder. Medicine (Baltimore) 95, 2473 (2016).

8 Ingelman-Sundberg M, Sim SC, Gomez A et al. Influence of cytochrome $\mathrm{P} 450$ polymorphisms on drug therapies: pharmacogenetic, pharmacoepigenetic and clinical aspects. Pharmacol. Ther. 116, 496-526 (2007).

9 Zhou SF. Polymorphism of human cytochrome P450 2D6 and its clinical significance: part I. Clin. Pharmacokinet. 48, 689-723 (2009).

10 Gardiner SJ, Begg EJ. Pharmacogenetics, drug-metabolizing enzymes, and clinical practice. Pharm. Rev. 58, 521-590 (2006). treated with SSRIs, especially fluoxetine or paroxetine, or other CYP2D6 substrates and inhibitors.

\section{Financial \& competing interests disclosure}

The authors have no relevant affiliations or financial involvement with any organization or entity with a financial interest in or financial conflict with the subject matter or materials discussed in the manuscript. This includes employment, consultancies, honoraria, stock ownership or options, expert testimony, grants or patents received or pending, or royalties.

No writing assistance was utilized in the production of this manuscript.

11 The Human Cytochrome P450 (CYP) Allele Nomenclature Database. www.cypalleles.ki.se/

12 Gaedigk A. Complexities of CYP2D6 gene analyses and interpretation. Int. Rev. Psychiatry 25, 534-553 (2013).

13 Hicks JK, Bishop JR, Sangkuhl K et al. Clinical Pharmacogenetics Implementation Consortium (CPIC) Guideline for CYP2D6 and CYP2C19 genotypes and dosing of selective serotonin reuptake inhibitors. Clin. Pharmacol. Ther. 98, 127-134 (2015).

14 Gaedigk A, Sangkuhl K, Whirl-Carillo M, Klein TE, Leeder JS. Prediction of CYP2D 6 phenotype from genotype across world populations. Genet. Med. doi:10.1038/gim.2016.80 (2016) (Epub ahead of print).

15 Bradford LD. CYP2D6 allele frequency in European Caucasians, Asians, Africans and their descendants. Pharmacogenomics 3, 229-243 (2002).

16 Solus JF, Arietta BJ, Harris JR et al. Genetic variation in eleven Phase I drug metabolism genes in an ethnically diverse population. Pharmacogenomics 5, 895-931 (2004).

17 Preissner S, Kroll K, Dunkel M et al. SuperCYP: a comprehensive database on Cytochrome P450 enzymes including a tool for analysis of CYP-drug interactions. Nucleic Acids Res. 38, D237-D243 (2010).

18 Jurica J, Zourkova A. Dynamics and persistence of CYP2D6 inhibition by paroxetine. J. Clin. Pharm. Ther. 38, 294-300 (2013).

19 Zourkova A, Hadasova E. Paroxetine-induced conversion of cytochrome P450 2D6 phenotype and occurence of adverse effects. Gen. Physiol. Biophys. 22, 103-113 (2003).

20 de Leon J. Pharmacogenetic tests in psychiatry. From fear to failure to hype. J. Clin. Psychopharm. 36, 1-6 (2016).

21 Isidoro-García M, Sánchez-Martín A, García-Berrocal B, Román-Curto C. Primun non nocere, polypharmacy and pharmacogenetics. Pharmacogenomics 16, 1903-1905 (2015). 